\title{
Point diffraction interferometry to measure local curvatures and caustics of noisy wave fronts: Application for determining optical properties of fish lenses
}

S. Vallmitjana

I. Ricart

Isaac.ricart.bou@gmail.com

S. Bosch

sbosch@ub.edu

\section{A. Gargallo \\ ana.gargallo@usc.es \\ E. Acosta \\ eva.acosta@usc.es}

\author{
Departament de Física Aplicada i Òptica, Facultat de Física, Universidad de Barcelona, 08028 \\ Barcelona, Spain \\ Departament de Física Aplicada i Òptica, Facultat de Física, Universidad de Barcelona, 08028 \\ Barcelona, Spain \\ Departament de Física Aplicada i Òptica, Facultat de Física, Universidad de Barcelona, 08028 \\ Barcelona, Spain \\ Departamento de Física Aplicada, Facultad de Física, Universidad de Santiago de Compostela, 15782 \\ Santiago de Compostela, Spain \\ Departamento de Física Aplicada, Facultad de Física, Universidad de Santiago de Compostela, 15782 \\ Santiago de Compostela, Spain
}

The study of caustics is important because they contain information about the image formation properties of optical systems. In this work we use the concept of caustic as a set of focal points, and we have developed a second order approach theory to determine local slopes and curvatures of a wavefront emerging from an optical system. The method is based on the use of a point diffraction interferometer, and the analysis of the interferograms allows us to compute the focal region. Experimental results obtained with a plano-convex lens demonstrate the accuracy of the combined theoretical-experimental method here developed. Application to noisy wavefronts such as those produced by biological samples, specifically in crystalline lenses of fish eyes, are also exposed.

[DOI: http://dx.doi.org/10.2971/jeos.2015.15010]

Keywords: Caustics, wavefront curvatures, point difraction interferometry, noisy wavefronts, fish lenses

\section{INTRODUCTION}

Caustics have been subject of research for many years. They encode information about the image formation properties of an optical system and thus they have been used among many other applications to test mirrors, to compute the disk of least confusion, to design null screens and to optimize optical systems [1]-[3]. Caustics have been studied from different perspectives. For instance, Stavroudis [4] developed a general integral of the eikonal equation in the particular case of a homogeneous optical medium and Burkhard and Shealy [5] have computed the caustic surfaces as the locus of singularities of the flux density of the emanating radiation. Caustics can be defined as the envelope of a system of orthotomic rays, and also as the locus of the principal centers of curvature of a wavefront. Here we will use the concept of caustic as a set of focal points, and by means of a local second order analysis we will derive a simple method to compute them.

The spherical crystalline lenses in the eyes of many fish species are well-suited models for studies on how natural selection has influenced the evolution of the optical system [6]. As in all vertebrates, fish eyes are similar to a photographic camera. In eye lenses the cornea and the crystalline lens provide a sharp image in the retina. Nevertheless, for fish lenses where the cornea is surrounded by water and both refractive indices are quite similar, focusing light on the retina is mainly the task of the crystalline lens [7]. Among fish, the lenses of teleosts have received the most attention, not only because of easy access to fresh material and the simple geometry of the lenses, which are typically spherical (hence they have only spherical aberrations) [8]-[10] but also because chromatic defocus is compensated for by what is called multifocality of the lens.

Multifocality can be quantified as the longitudinal spherical aberration curve of the lens. Such curves for different species are usually obtained by scanning a thin laser beam through a meridional plane of the lens and analysis of the beam paths [11, 12]. Many biological multifocal optical systems have not yet been fully explored or understood and this is due in part to the insufficient accuracy of the experimental methods used. We present here a new interferometric method that provides at the same time the slopes and the local curvatures of the phase of a non-paraxial beam even under noisy conditions such as those presented in biological samples. The theoretical approach to evaluate not only the longitudinal spherical aberration but also the location of caustics is also presented. The accuracy of the method is shown by numerical simulations and experimental calibration by means of a plano-spherical glass ball. The method is applied to evaluate the optical properties of a teleost fish eye lens (Scorpaena Scrofa).

\section{THEORY}

Any continuous function three times differentiable (i.e, including polynomials of any order) can be locally approxi- 


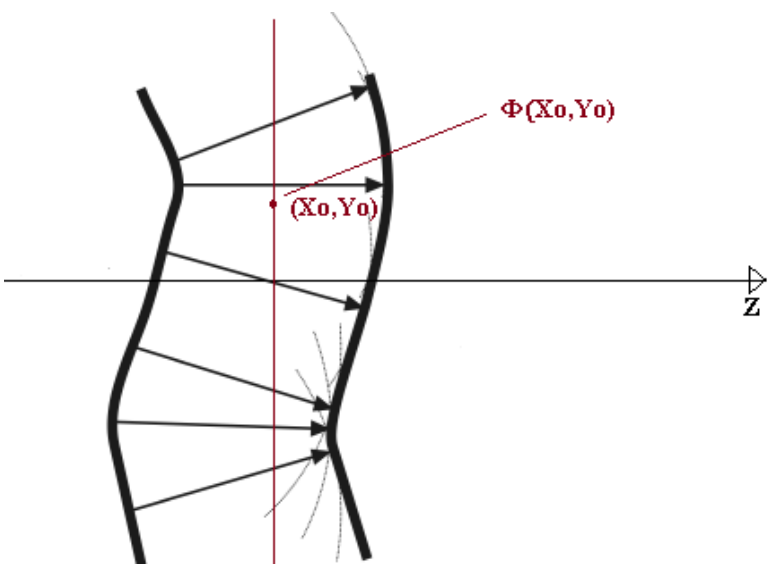

FIC. 1 Terminology used for an optical beam propagating in free space.

mated around a point to a second order polynomial (Taylor theorem). The region around the point where the approximation is 'good' depends on the third order derivatives of the function, which determine the approximation error of the function to a second order polynomial [13]. Thus let $\Phi(x, y, z)$ be the phase of an optical beam propagating in free space. For a preferred propagation direction, named $z$, and in the vicinities of a point $\left(x_{0}, y_{0}\right)$ of a plane $(z=0)$ perpendicular to the propagation direction as shown in Figure 1, the phase of a beam can be approximated by the second order Taylor expansion as:

$$
\begin{aligned}
\Phi(x, y) & \approx \Phi x_{0}, y_{0}+\left.\frac{\partial \Phi}{\partial x}\right|_{x_{0}, y_{0}}\left(x-x_{0}\right)+\left.\frac{\partial \Phi}{\partial y}\right|_{x_{0}, y_{0}}\left(y-y_{0}\right) \\
& +\frac{1}{2}\left[\left.\frac{\partial^{2} \Phi}{\partial x^{2}}\right|_{x_{0}, y_{0}}\left(x-x_{0}\right)^{2}+\left.2 \frac{\partial^{2} \Phi}{\partial x \partial y}\right|_{x_{0}, y_{0}}\left(x-x_{0}\right)\left(y-y_{0}\right)\right. \\
& \left.+\left.\frac{\partial^{2} \Phi}{\partial y^{2}}\right|_{x_{0}, y_{0}}\left(y-y_{0}\right)^{2}\right]
\end{aligned}
$$

For a non-paraxial beam $\left.\frac{\partial \Phi}{\partial x}\right|_{x_{0}, y_{0}}=\sin \alpha_{0}$ and $\left.\frac{\partial \Phi}{\partial y}\right|_{x_{0}, y_{0}}=$ $\sin \beta_{0}$ represent the direction cosines of the rays at the chosen plane and the Hessian of $\Phi(x, y)$ expressed in a matrix form as

$$
\left(\begin{array}{ll}
\left.\frac{\partial^{2} \Phi}{\partial x^{2}}\right|_{x_{0}, y_{0}} & \left.\frac{\partial^{2} \Phi}{\partial x \partial y}\right|_{x_{0}, y_{0}} \\
\left.\frac{\partial^{2} \Phi}{\partial x \partial y}\right|_{x_{0}, y_{0}} & \left.\frac{\partial^{2} \Phi}{\partial y^{2}}\right|_{x_{0}, y_{0}}
\end{array}\right)
$$

represents the curvatures of the phase at the point $\left(x_{0}, y_{0}\right)[14]$.

For a rotationally symmetric beam the optical properties can be studied in the tangential plane $(X=0)$ and Eq. (1), neglecting the constant phase (piston) reduces to

$$
\begin{aligned}
\Phi(x, y) \approx & \left.\frac{\partial \Phi}{\partial y}\right|_{x_{0}, y_{0}}\left(y-y_{0}\right)+\frac{1}{2}\left[\left.\frac{\partial^{2} \Phi}{\partial x^{2}}\right|_{x_{0}, y_{0}} x^{2}\right. \\
& \left.+\left.\frac{\partial^{2} \Phi}{\partial y^{2}}\right|_{x_{0}, y_{0}}\left(y-y_{0}\right)^{2}\right]
\end{aligned}
$$

For non-paraxial beams, i.e., when the direction cosines cannot be approximated by the angles themselves or the tangents Eq. (3) represents the phase of an astigmatic wavefront that

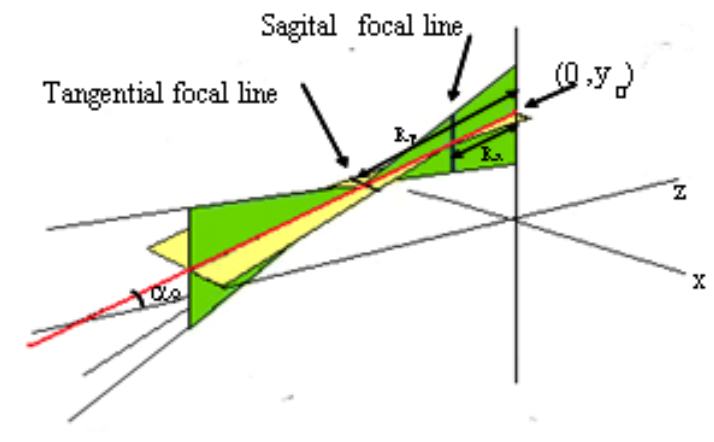

FIG. 2 Ray tracing equivalent.

we can rewrite as:

$$
\begin{aligned}
\Phi(x, y) \approx & \frac{2 \pi}{\lambda} \sin \alpha_{0}\left(y-y_{0}\right) \\
& +\frac{\pi}{\lambda}\left[\frac{x^{2}}{R_{x}}+\frac{\cos ^{2} \alpha_{0}\left(y-y_{0}\right)^{2}}{R_{y}}\right]
\end{aligned}
$$

Therefore $R_{x}$ and $R_{y}$ provide, respectively, the distance from $\left(x_{0}, y_{0}\right)$ to the sagittal focal line and the tangential focal line along the ray with direction cosine $\sin \alpha_{0}=\left.\frac{\partial \Phi}{\partial y}\right|_{x_{0}, y_{0}}$, passing through $\left(x_{0}, y_{0}\right)$. From a geometrical point of view we can represent this astigmatic wave as a narrow pencil beam of rays as shown in Figure 2, where it can be seen that $\alpha_{0}$ accounts therefore for the oblique incidence of the ray.

In the limit, when the size of the region about $\left(x_{0}, y_{0}\right)$ tends to zero, the focal lines become two focal points and the caustic surfaces of the wavefront can be regarded as the locus of the focal points. We will call them "approximated caustic" surfaces.

\section{EXPERIMENTAL METHOD}

\subsection{Principles}

The main element of a PDI is a semitransparent plate with a clear pinhole, as shown in Figure 3. Our semitransparent plate was made by coating a glass substrate with $\mathrm{Cr}$. The optical density was 2.5 and the pinhole diameter was 15 microns.

When a beam reaches the plate, a spherical reference wave is produced by diffraction at the clear pinhole while the rest of the beam passes through the plate without any change in phase. If the size of the pinhole and the transmittance of the plate are chosen in such a way that both beams have similar amplitude, well contrasted fringes will be observed in any plane placed after the plate. The theoretical basis of the PDI and some applications can be found in references [15]-[18].

In a plane at a distance $D$ from the pinhole (placed at $\left(x_{p}, y_{p}\right)$ position), the observed interference pattern corresponds to that produced by a spherical beam with focus at the pinhole and the phase of the incident beam at the observation plane. Thus, the fringe pattern provides information about the phase of the incident wave, with the interference term given by

$$
\Delta(x, y)=\left\{\Phi(x, y)-\frac{2 \pi}{\lambda} \sqrt{\left(x-x_{p}\right)^{2}+\left(y-y_{p}\right)^{2}+(D)^{2}}\right\}
$$




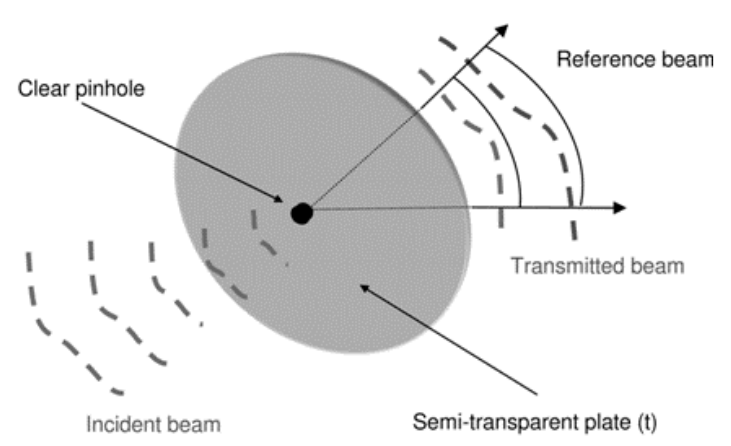

FIC. 3 Basic principle of PDI.

If the paraxial focus of the input beam does not lie in the pinhole of the PDI plate (when $x_{p} \neq 0, y_{p} \neq 0$ ) then linear carrier fringes are introduced in the interferograms. Spherical carrier fringes are introduced when the beam focuses either before or after the pinhole plate. On the other hand, the intensity distribution corresponding to the diffracted spherical beam is modulated by a Jinc function [18] and the more defocused the beam is, the smaller the region of well contrasted fringes becomes. Moreover, when $x_{p} \neq 0$ and/or $y_{p} \neq 0$ the region of visible fringes can be moved within the observation plane $[16,18]$. In this way the size and position of the region of well contrasted fringes can be chosen. Therefore, for high defocused incident beams, a few elliptical concentric fringes within a very small region of the observation plane can be recorded. We must point out here that if visible fringes are not elliptic, second order approach does not apply, therefore the pinhole must be moved in such a way that fringes become smaller and elliptical to ensure they are within the region where the approximating remainder term (as stated by Taylor theorems) is negligible.

For rotationally symmetric beams we can get all 3-D information by setting $x_{p}=0$ and varying only $y_{p}$. In this case, the interference patterns correspond to the interference of the local second order approaches of both the reference beam [13].

$$
\begin{aligned}
\Phi_{\mathrm{ref}}(x, y)= & \frac{2 \pi}{\lambda} \sqrt{\left(x-x_{p}\right)^{2}+\left(y-y_{p}\right)^{2}+(D)^{2}} \\
\approx & \frac{2 \pi}{\lambda} \sin \alpha_{0}\left(y-y_{0}-y_{p}\right) \\
& +\frac{\pi}{\lambda}\left[\frac{x^{2}}{\sqrt{D^{2}+\left(y_{0}-y_{p}\right)^{2}}}\right. \\
& \left.+\frac{D^{2}\left(y-\left(y_{0}-y_{p}\right)\right)^{2}}{\sqrt{\left(D^{2}+\left(y_{0}-y_{p}\right)^{2}\right)^{3}}}\right]
\end{aligned}
$$

and the beam under test [14]

$$
\begin{aligned}
\Phi(x, y) \approx & \frac{2 \pi}{\lambda} \sin \alpha_{0}\left(y-y_{0}\right) \\
& +\frac{\pi}{\lambda}\left[\frac{x^{2}}{R_{x}}+\frac{\cos ^{2} \alpha_{0}\left(y-y_{0}\right)^{2}}{R_{y}}\right]
\end{aligned}
$$

The center of the ellipses lies at the intersection point (on the observation plane) of the ray passing through the pinhole,

$$
\sin \alpha_{0}=\frac{\left(y_{0}-y_{p}\right)}{\sqrt{D^{2}+\left(y_{0}-y_{p}\right)^{2}}}
$$

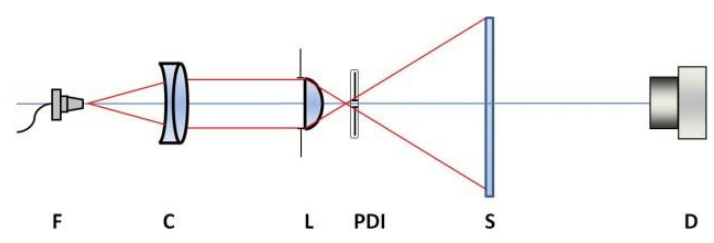

FIG. 4 Experimental set up for testing a plano-convex lens.

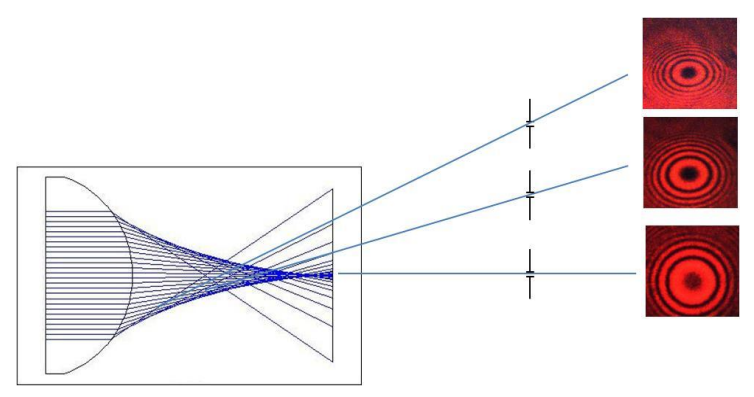

FIG. 5 Scanning of the phase at the observation plane by moving the pinhole transversally.

and it is common for both beams due to the fact that the center of the ellipses $\left(0, y_{0}\right)$ and the tangential and sagittal focus for both astigmatic beams lie in the same ray.

In practice, by controlling the pinhole position $y_{0}$ and by knowing the distance $D, \sin \alpha_{0}$ can be measured. From the measurement of the major and minor axis of any of the interference fringes (maxima or minima) $R_{x}$ and $R_{y}$ are straightforward to evaluate.

In short, the interferometer can perform a scanning of the beam in the observation plane providing first and second order derivatives of the beam as in Eq. (3) or equivalently the local slopes 0 , and the local curvatures $R_{x}$ and $R_{y}$ as in Eq. (4).

\subsection{Accuracy of the method}

In order to demonstrate experimentally the accuracy of both the quadratic approach as well as the interferometer, on one hand we developed the experimental set up shown in figure 4 to determine the caustics position of a beam produced by a plano-convex lens illuminated by an on-axis plane wave. $\mathrm{F}$ is a monomode optical fiber connected to a He-Ne laser (@633 nm), C is a collimating lens, L is the lens under test (Thorlabs, LA1951), PDI is the point diffraction interferometer, $S$ is a screen and D is a CCD camera.

In order to obtain what we call "experimental caustic" we fit the first dark fringe of the interferograms to an ellipse in order to obtain the values for the major and minor axis and therefore $R_{x}$ and $R_{y}$. From the height of the pinhole, the center of the ellipses $y_{0}$ and the distance from the pinhole to the observation plane we calculate ${ }_{0}$. Due to the symmetry the scanning is performed for only positive or negative values of $y_{p}$. Figure 5 illustrates more clearly the relationship between the pinhole position and the center of the ellipses as explained above. 


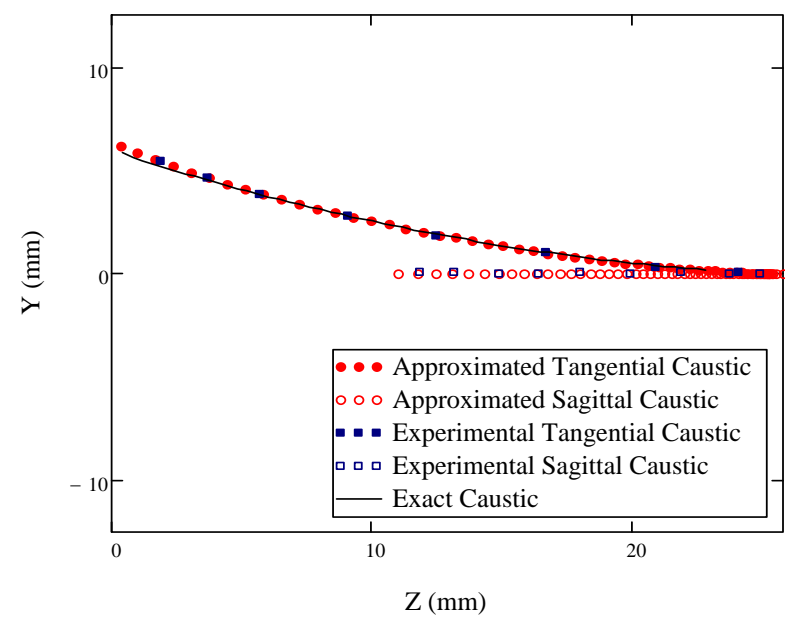

FIG. 6 Accuracy of the caustics position determination. Approximated: computed by numerical evaluation of the first and second derivatives of the phase. Experimental: from the measured values of the semi-axis of the ellipses in the interferograms. Exact: obtained by ray tracing as described in [19].

On the other hand the optical path length (OPL) of the rays at the observation plane $S$ is calculated by ray tracing [19] and values are fitted to an $8^{\text {th }}$ degree polynomial in the radial coordinate. From the fit, the first and second order local partial derivatives of the OPL are evaluated to obtain numerical values for $\alpha_{0}, R_{x}$ and $R_{y}$ respectively and hence the locus of what we call "approximated caustics". Moreover the exact position of caustics for the lens can also be numerically determined by ray tracing, as described in Reference [19]. This is the so called 'exact' caustic. Figure 6 summarizes the results. We can see that the second order approach for the numerical determination of the caustics position as well as for the experimental one are in good agreement with the exact position of the caustics surfaces for a wavefront that is well approximated by an $8^{\text {th }}$ degree polynomial or, equivalently, in this case with an $8^{\text {th }}$ order spherical aberration. As could be expected, as the height of the marginal ray increases, the accuracy of the approximation decreases. Nevertheless, as we will see in next section, this accuracy is enough to calculate caustics surfaces for eye lenses.

\section{OPTICAL PROPERTIES OF FISH LENSES}

An eye lens of a scorpion fish was placed in a glass cell filled with supplemented Medium 199 plus solution to prevent fast degeneration. The lens was excised a few hours postmortem. We used the same set up as that in Figure 4 but replacing the plano-convex by the eye lens in the cell as shown in Figure 7.

Figure 8 shows the fringes at the observation plane and the close up image used to fit the first dark fringe to an ellipse. It can be seen that fringes are much noisier than those for the plano-convex lens, with an acceptable contrast, though.

As in the previous section the PDI plate is displaced vertically to allow scanning of the local curvatures and the slope of the phase across the observation plane. From the size of the axes of the first dark ellipse, the local curvatures (or equivalently

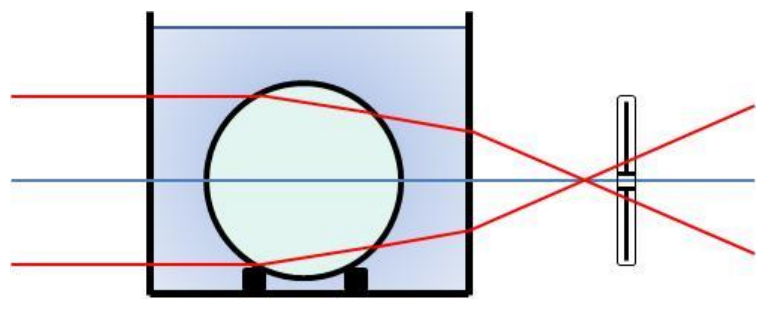

FIG. 7 Detail of the set up for the measurement of eye lenses. Glass cell with the lens illuminated by a plane wave and PDI plate in the vicinities of the focal region of the fish eye lens.

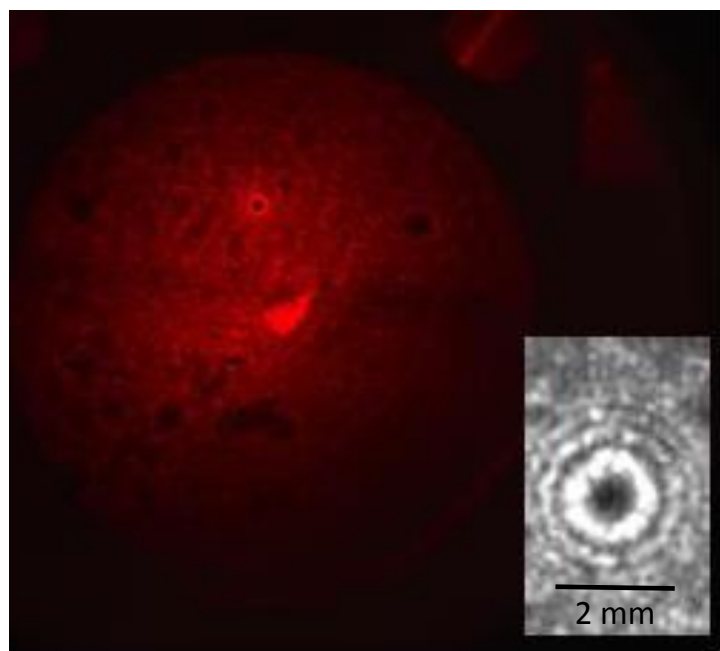

FIG. 8 Noisy interferograms for a fish lens.

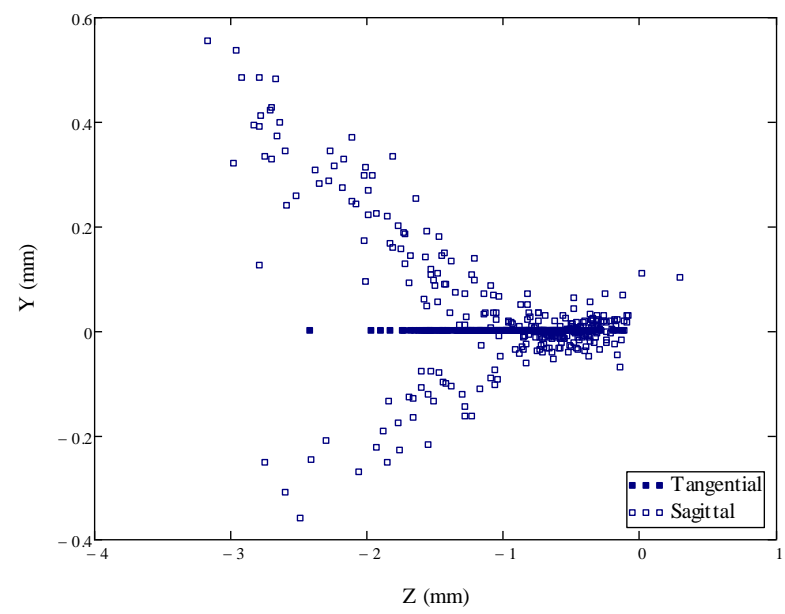

FIG. 9 Tangential and sagittal caustics of fish crystalline lens.

$R_{x}$ and $R_{y}$ ) are obtained, and the slope is determined by controlling the position of the pinhole. In this case, in order to check the symmetry of the lens, the scanning is done to obtain both upper and lower branches of the tangential caustic surface. With all these data, the position of the corresponding points at the caustic surfaces is evaluated as explained in Section 3.1. Figure 9 shows the results for tangential and sagittal caustics.

We can observe that the position of the caustic surfaces is not 
as smooth as in the plano-convex lens. Nevertheless the presence of spherical aberration of the eye lens is notorious. The symmetry is not perfect but there are no significative variations between the upper and the lower parts of the caustic surface. The experimental data for the caustics can be fitted to find a smooth curve describing the main aberration as well as to quantify the noise of this type of biological tissues. The multifocality for different wavelengths can be directly evaluated.

\section{CONCLUSIONS}

We can therefore perform a mapping of noisy wavefronts and obtain the caustic surfaces by means of a second order approach of wavefronts and a point diffraction interferometer. We have obtained simple formulae to locate caustics in the tangential plane, as a proof of concept that a simple second order approach of the phase in a plane can provide accurate location of the caustic surfaces. We believe that the method for obtaining the caustic reported here is easy, conceptually simple and fast to implement. The study of caustics in 3D, with no rotational symmetry, will be subject of a future work. This mapping of the phase is of use for highly aberrated beams where the dynamic range of interferometers is not sufficient just like those produced by some ophthalmic lenses [12, 16] or, as we have shown here, for noisy wavefronts where noise can either destroy coherence or induce phase dislocation in the interferograms and therefore invalidate measurements as is the case of some biological samples such as fish eyes.

\section{ACKNOWLEDGEMENTS}

This work was supported by the Spanish Ministerio de Economia y Competitividad, grant FIS2012-38244 and Xunta de Galicia, grant CN 2012/156.

\section{References}

[1] D. Malacara, Optical shop testing (Wiley, New Jersey, 2007).

[2] M. Avendaño-Alejo, "Caustics in a meridional plane produced by plano-convex aspheric lenses," J. Opt. Soc. Am. A 30(3), 501-508 (2013).

[3] I. H. Al-Ahdali, and D. L. Shealy, "Optimization of three- and fourelement lens systems by minimizing the caustic surfaces," Appl. Optics 29(31), 4551-4559 (1990).
[4] 0. N. Stavroudis, The Mathematics of Geometrical and Physical Optics: the $k$-function and its Ramifications (Wiley-VCH, Weinheim, 2006).

[5] D. G. Burkhard, and D. Shealy, "Formula for the density of tangent rays over a caustic surface," Appl. Optics 21, 3299-3306 (1982).

[6] M. F. Land, and D. E. Nilsson, Animal Eyes (Oxford University Press Inc., New York, 2002).

[7] T. Mandelman, and J. G. Sivak, "Longitudinal chromatic aberration of the vertebrate eye," Vision Res. 23, 1555-1559 (1983).

[8] G. L. Walls, The Vertebrate Eye and its Adaptive Radiation (Cranbrook Institute of Science, Bloomfield Hills, 1964).

[9] R. H. H. Kröger, K. A. Fritsches, and E. J. Warrant, "Lens optical properties in the eyes of large marine predatory Teleosts," J. Comp. Physiol. A 195, 175-182 (2009).

[10] R. H. H. Kröger, "Multifocal Optics of the Fish Lens for Clear Color Vision," in Frontiers in Optics 2011, FWV1 (Optical Society of America, San Jose, 2011).

[11] M. C. W. Campbell, and A. Hughes, "An analytic, gradient index schematic lens and eye for the rat which predicts aberrations for finite pupils," Vision Res. 21, 1129-1148 (1981).

[12] J. G. Sivak, "Optical properties of a cephalopod eye (the short finned squid, Illex illecebrosus)," J. Comp. Physiol. A 147, 323-327 (1982).

[13] J. E. Marsden, and A. J. Tromba, Vector calculus, 2nd ed. (W. H. FreemanctCo., S. Francisco, 1981).

[14] C. Campbell, "Ceneralized Coddington equations found via an operator method," J. Opt. Soc. Am. A 23(7), 1691-1697 (2006).

[15] V. Linikk, "Simple interferometer for the investigation of optical systems," C. R. Acad. Sci. USSR 1, 208-210 (1933).

[16] S. Chamadoira, R. Blendowske, and E. Acosta, "Progressive Addition Lens Measurement by Point Diffraction Interferometry," Optometry Vision Sci. textbf89(10), 1532-1542, (2012).

[17] E. Acosta, S. Chamadoira, and R. Blendowske, "Modified point diffraction interferometer for inspection and evaluation of ophthalmic components," J. Opt. Soc. Am. A. 23, 632-637 (2006).

[18] S. Chamadoira, Point diffraction interferometer for inspection and characterization of progressive addition lenses, Ph.D. Dissertation (University of Santiago de Compostela, Santiago de Compostela, 2011)

[19] S. Bosch, "Ray tracing and scalar diffraction calculations of wavefronts, caustics and complex amplitudes in optical systems," J. Mod. Optic. 58, 146-153 (2011). 